\title{
Moving forward with fuel cells
}

\author{
For hydrogen to make a greater impact in our energy systems, attention is required on the integration of new \\ catalysts into fuel cells and their needs in emerging applications, such as heavy-duty transport.
}

f you knew where to look, it never really went away. Yet it's hard to escape the feeling that hydrogen is firmly back on the energy agenda. The EU's Hydrogen Strategy ${ }^{1}$ placed the gas at the heart of their energy and climate plans. Similarly, low-cost carbon-free hydrogen is a goal of the Biden administration's recent climate innovation initiatives $^{2}$. There is strong support for hydrogen in China and elsewhere, too ${ }^{3}$.

One of hydrogen's strengths is its versatility. It can be combusted to generate heat or fed into fuel cells, along with oxygen, to produce electrical power directly. It can act as an energy storage medium via electrolysis of water using excess electricity. It can also play a role in hard-to-decarbonize sectors, such as heating and cement and steel making. Its high gravimetric energy density also makes it attractive in transportation.

In recent decades, light-duty passenger vehicles have been the main applications focus for hydrogen-powered transportation. Commercialized light-duty vehicles that run on hydrogen are powered by polymer electrolyte membrane fuel cells (PEMFCs). At the core of a PEMFC sits a membrane electrode assembly (MEA): a polymer membrane sandwiched between two electrodes. At the anode, hydrogen is oxidized to protons, while at the cathode, oxygen is reduced.

PEMFCs typically use platinum-based catalysts to facilitate the oxygen reduction reaction. Given its high price, immense efforts have sought to minimize the amount of platinum used without sacrificing performance. Catalyst design strategies have included alloying platinum with cheaper metals and tailoring the nanostructure of catalytic particles.

Much of the progress in catalyst design has been achieved through half-cell measurements in aqueous solutions using the thin-film rotating disk electrode (RDE) technique. Countless catalysts with exceptional activities on a mass basis have been reported thus. However, while RDE measurements are valuable to screen catalysts and to develop structure-property relationships, they do not accurately replicate real fuel cell conditions. Highly performing catalysts as observed by RDE measurements have not necessarily translated into similarly impressive performance in a MEA.
In a Review in this issue, $\mathrm{Li}$ and colleagues explore the reasons for this disconnect and strategies to bridge the gap. A key factor is the large difference in operating current density between the two systems: MEAs often operate at $>1 \mathrm{~A} \mathrm{~cm}^{-2}$, compared to $\leq 10 \mathrm{~mA} \mathrm{~cm}^{-2}$ for RDE measurements. Moving from RDE to MEA measurements changes the proton and oxygen transport mechanisms at play.

To address this, Li and colleagues suggest a stronger focus on the MEA's catalyst layer and on approaches to improve the local oxygen mass transport within it. The key constituents of a catalyst layer are the catalyst particles, a support and an ion-conducting polymer (ionomer). The interactions between these three components are particularly important to optimize. The researchers also suggest making more use of intermediate testing procedures, between RDE and MEA testing.

Enhancing PEMFC performance, while decreasing costs, would improve their viability for light-duty vehicles. Yet, this is unlikely to be enough on its own - a lack of refuelling infrastructure has also hampered widespread adoption, while battery electric vehicles (BEVs) have made exceptional headway.

The danger for hydrogen is that a move to BEVs is well underway: hydrogen-fuelled approaches could be shut out of the light-duty vehicle market. BEVs appear to have greater manufacturer buy-in and they - and their charging infrastructure - are increasingly common on roads. Meanwhile, fuel cell vehicles (FCVs) remain more rare, despite their strengths of greater driving range and rapid refuelling.

Yet there is increasing interest in hydrogen's role in decarbonizing heavy-duty and long-haul transportation; for example, heavy goods vehicles, buses, rail, marine and aviation. Compared to Li-ion batteries, fuel cell systems scale more attractively to meet the high energy and power demands of heavy-duty vehicles (HDVs): increasing the size of fuel cell stacks and hydrogen tanks results in a lower weight penalty than is suffered using batteries. Moreover, HDVs typically have better defined transit routes than, say, passenger vehicles, meaning that refuelling can be more easily planned.

Kusoglu and colleagues pick up this subject in their Review. They consider the emerging markets for FCVs in the context of hydrogen roadmaps from around the globe. They highlight that while HDV applications present substantive opportunities for fuel cells, they also introduce distinct demands on the technology.

In particular, moving to HDVs shifts the balance from capital to operating costs, making extremely low platinum loadings at the electrodes less critical. On the other hand, higher cell voltages will be needed to achieve optimal efficiency and greater driving distances will necessitate longer fuel cell lifetimes. Although learning from light-duty vehicles can inform the development of HDV fuel cells, research needs to address the different efficiency and durability requirements, which impose critical system and materials challenges.

Kusoglu and colleagues comment that early electric HDVs may be battery powered in part due to recharging infrastructure already being in place. However, with time, they believe fuel cell HDVs may emerge due to their inherent advantages, including the separation of energy storage and power output. Yet it need not necessarily be a case of either/or: batteries and fuel cells can be considered as complementary approaches. The recent US Department of Energy call to decarbonize the trucking sector SuperTruck 3 - specifically calls out hybrid strategies, such as batteries plus fuel cell range extenders, as a point of focus ${ }^{4}$.

Increased commitments to decarbonize transport and heating provide renewed hope for the gas to play a pivotal role in the near future. A key part of its success will be fuel cells that are ever cheaper and more highly performing. To achieve that, successful integration of catalysts into fuel cells is crucial, as is designing devices holistically to meet the demands of specific applications. $\square$

Published online: 21 May 2021

https://doi.org/10.1038/s41560-021-00846-1

References

1. A Hydrogen Strategy for A Climate-Neutral Europe (European Commission, 2020).

2. Biden-Harris administration launches American innovation effort to create jobs and tackle the climate crisis. The White House https://go.nature.com/3us22w0 (2021).

3. The hydrogen stream: projects move forward in China, Japan, Australia and across several European countries. PV magazine https://go.nature.com/3nU7BAT (2021).

4. DOE announces $\$ 162$ million to decarbonize cars and trucks. US Department of Energy https://go.nature.com/3xUj17p (2021). 